STRUCTURAL SCIENCE CRYSTAL ENGINEERING MATERIALS

ISSN 2052-5206

Received 18 August 2020

Accepted 10 September 2020

Edited by A. J. Blake, University of Nottingham, United Kingdom

Keywords: martensites; phase transitions; group theory; Heusler alloys; order parameters.

\section{Fundamental aspects of symmetry and order parameter coupling for martensitic transition sequences in Heusler alloys. Erratum}

\author{
Michael A. Carpenter ${ }^{\mathrm{a} *}$ and Christopher J. Howard ${ }^{\mathrm{b}}$
}

${ }^{\mathbf{a}}$ Department of Earth Sciences, University of Cambridge, Downing Street, Cambridge CB2 3EQ, United Kingdom, and
$\mathbf{b}_{\text {b }}$ chool of Engineering, University of Newcastle, Callaghan, NSW 2308, Australia. *Correspondence e-mail:
mc43@esc.cam.ac.uk

In the course of further studies of phase transitions in martensites [Driver, Salje, Howard, Lampronti, Ding \& Carpenter (2020), Phys. Rev. B, 102, 014105], errors were uncovered in a few entries in Table 3 of the paper by Carpenter \& Howard [(2018), Acta Cryst. B74, 560-573]. The required corrections are given here.

In the course of detailed studies of the phase transitions in $\mathrm{Ti}_{50} \mathrm{Pd}_{50-; x} \mathrm{Cr}_{x}$ martensites (Driver et al., 2020), we found there were errors in some entries in Table 3 in the paper by Carpenter \& Howard (2018):

(i) The dimensions of the rhombohedral cell associated with space group $P \overline{3}$, recorded in the second last column, should be amended to read $3 a_{0}, 3 a_{0}, 3 a_{0}$. This cell is a smaller one than that shown in the table and the rhombohedral angle in it is close to $90^{\circ}$.

(ii) A number of corrections need to be made in the column headed 'Other labels', the final column in this table.

(a) The entry ' $3 \mathrm{R}$ or $2 \mathrm{M}$ ' at the first appearance of space group $P 2 / m$ is to be removed.

(b) The entry ' $9 \mathrm{R}$ or $6 \mathrm{M}$ ' at the second appearance of space group $P 2 / m$ is to be replaced by ' $3 \mathrm{R}$ or $2 \mathrm{M}$ '.

The label ' $9 \mathrm{R}$ or $6 \mathrm{M}$ ' would be associated with space group $P 2 / m$ at $\mathbf{k}=\frac{1}{9}, \frac{1}{9}, 0$, although the table as it was printed does not extend to this.

The 'Other labels' were based largely on the work of Otsuka et al. (1993). We wish to comment briefly on the matter of notation, with particular reference to Fig. 4 in the Otsuka paper. The different martensite structures are shown as being based on different stackings of the nearly close-packed (110) planes of the parent B2 structure, space group $\operatorname{P} m \overline{3} m$. There are considered to be three different stacking positions, A, B and $\mathrm{C}$. It can be seen in Otsuka's figure that these positions are separated by the translation vector $(\overline{1}, 1,0) / 3$ - since the first basis vector for the monoclinic structures is $(\overline{1}, 1,0)$ [Table 3 in Carpenter \& Howard (2018)], this vector is just $\mathbf{a}_{m} / 3$. We now consider the Zhdanov symbols (Zhdanov, 1945) which describe the stacking sequence, using +1 for 'clockwise' stacking such as A followed by B, and -1 for the reverse 'anticlockwise' stacking. In this notation the symbol $(m \bar{n})$ indicates that there are $m$ stackings of a clockwise nature (starting from a zero-th layer) followed by $n$ anticlockwise stackings. The stacking sequence in Otsuka's Fig. $4(c)$ is 
Table 1

Comparison of the B2-based structures from Table 1 in Otsuka et al. (1993) with the corresponding theoretical structures taken from Table 3 of Carpenter \& Howard (2018).

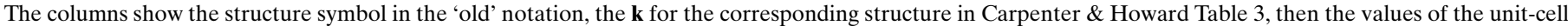

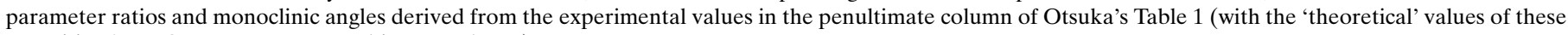
quantities from Carpenter \& Howard in parentheses).

\begin{tabular}{|c|c|c|c|c|}
\hline 'Old' notation from Otsuka et al. & Carpenter \& Howard $\mathbf{k}$ & $a / b$ & $c / a$ & $\beta\left({ }^{\circ}\right)$ \\
\hline $2 \mathrm{H}$ & $\frac{1}{2}, 0, \frac{1}{2}$ & $1.542(1.414)$ & $0.981(1.000)$ & $90(90)$ \\
\hline $3 \mathrm{R}$ & $\frac{1}{3}, \frac{1}{3}, 0$ & $1.542(1.414)$ & $1.502(1.581)$ & $100(108)$ \\
\hline $7 \mathrm{R}$ & $\frac{1}{7}, \frac{1}{7}, 0$ & $1.551(1.414)$ & $3.464(3.536)$ & $94.4(98)$ \\
\hline 9R & $\frac{1}{9}, \frac{1}{9}, 0$ & $1.648(1.414)$ & $4.350(4.528)$ & $91.6(96)$ \\
\hline
\end{tabular}

This table includes calculated values for $\mathbf{k}=\frac{1}{9}, \frac{1}{9}, 0$, although they were not included in Table 3 of Carpenter \& Howard.

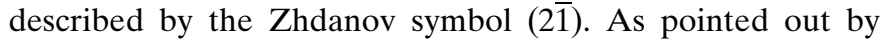
Delaey \& Chandrasekaran (1995), the structure can be described using a primitive monoclinic cell constructed from the first complete stacking sequence, in this case just $(2 \overline{1})$. Otsuka et al. prefer to describe structures on the $B$-centred monoclinic cell, and for this purpose they need two complete stacking sequences. In the case under consideration this is indicated by $(2 \overline{1})_{2}$. In order for the final layer to lie directly above the zero-th layer, the difference between the number of clockwise and the number of anticlockwise steps must be a multiple of 3. In this case, three complete stacking sequences are required, that is $(2 \overline{1})_{3}$. The numeral in the Ramsdell notation (Ramsdell, 1947) represents the number of layers required to have the final layer lie over the initial layer, in this case 9, leading to Ramsdell symbol 9R.

There is some consensus (Tadaki et al., 1975; Martynov et al., 1983; Ohba et al., 1990) that in practice the stacking fault shear can exceed (by up to $\sim 25 \%$ ) its ideal $\mathbf{a}_{m} / 3$. This means the final layer no longer lies exactly over the zero-th layer, the shift being manifested as a modest monoclinic distortion.

There remain questions as to the relationships between these different stacking sequences and the description according to harmonic displacement models implied by the entries in Carpenter \& Howard's Table 3. From its definition, a structure for which the Ramsdell symbol is $n \mathrm{R}$, seen as a stacking of (110) layers (i.e. viewed along the [110]* direction), should show a repeat after $n$ layers. Now the entry in Carpenter \& Howard's Table 3 with $\mathbf{k}=1 / n, 1 / n, 0$ corresponds to a pattern of transverse displacements of the (110) layers with a period of $n$ layers, matching in some sense the stacking sequence in the Ramsdell structure. For $n$ odd the final layer, strictly, does not lie directly over the initial layer [see Fig. 3(c) in Carpenter \& Howard], but leads to monoclinic distortion for the example of $n=7$ the monoclinic angle is (from Table 3 in Carpenter \& Howard) $98^{\circ}$. It is interesting to note that Noda et al. (1990) examined different models to describe the structure in the 7R martensite, and found harmonic models fitted their data better than the stacking-fault ones.

The relationship between the different stacking sequences and the entries in Carpenter \& Howard's Table 3 are best confirmed by comparing unit-cell parameter ratios and monoclinic angles from Carpenter \& Howard's Table 3 with the experimental values derived from Otsuka's Table 1; see Table 1 in the present paper.

The agreement evident in this table strongly supports the correspondences we have proposed. The higher value of $a / b$ compared with the theoretical value represents a distortion that makes the (110) layers more nearly close-packed (Otsuka et al., 1993). The monoclinic angles are, however, not so informative, because for $n$ odd they are determined, in the Carpenter \& Howard scheme, by a shift of $\mathbf{a}_{m} / 2$ between the zero-th and $n$th layers, whereas in the Ramsdell stacking-fault description the corresponding shift, even if non-zero, is unlikely to exceed this value.

\section{References}

Carpenter, M. A. \& Howard, C. J. (2018). Acta Cryst. B74, 560-573. Delaey, L. \& Chandrasekaran, M. (1995). J. Phys. IV Colloq. 05(C2), 251-256.

Driver, S. L., Salje, E. K. H., Howard, C. J., Lampronti, G. I., Ding, X. \& Carpenter, M. A. (2020). Phys. Rev. B, 102, 014105.

Martynov, V. V., Enami, K., Khandros, L. G., Nenno, S. \& Tkachenko, A. V. (1983). Phys. Met. Metall. 55, 136-143.

Noda, Y., Shapiro, S. M., Shirane, G., Yamada, Y. \& Tanner, L. E. (1990). Phys. Rev. B, 42, 10397-10404.

Ohba, T., Emura, Y., Miyazaki, S. \& Otsuka, K. (1990). Mater. Trans. Jpn. Inst. Met. 31, 12-17.

Otsuka, K., Ohba, T., Tokonami, M. \& Wayman, C. M. (1993). Scr. Metall. Mater. 29, 1359-1364.

Ramsdell, L. S. (1947). Am. Mineral. 32, 64-82.

Tadaki, T., Tokoro, M. \& Shimizu, K. (1975). Trans. Jpn. Inst. Met. 16, 285-296.

Zhdanov, G. S. (1945). C. R. Acad. Sci. USSR, 48, 39-42. 